\title{
Spectrum sharing in multi-channel cooperative cognitive radio networks: a coalitional game approach
}

\author{
Yu-Wei Chan $\cdot$ Feng-Tsun Chien $\cdot$ Ronald Y. Chang • \\ Min-Kuan Chang $\cdot$ Yeh-Ching Chung
}

(C) Springer Science+Business Media New York 2013

\begin{abstract}
In this paper, we study a coalitional game approach to resource allocation in a multi-channel cooperative cognitive radio network with multiple primary users (PUs) and secondary users (SUs). We propose to form the grand coalition by grouping all PUs and SUs in a set, where each PU can lease its spectrum to all SUs in a time-division manner while the SUs in return assist PUs' data transmission as relays. We use the solution concept of the core to analyze the stability of the grand coalition, and the
\end{abstract}

\section{Y.-W. Chan · Y.-C. Chung $(\bowtie)$}

Department of Computer Science, National Tsing Hua

University, SSLAB Rm 719, Department of Computer Science

101, Section 2 Kuang Fu Road, Hsinchu, Taiwan

e-mail: ychung@cs.nthu.edu.tw

Y.-W. Chan

e-mail: ywchan@sslab.cs.nthu.edu.tw

\section{F.-T. Chien}

Department of Electronics Engineering, National Chiao Tung

University, Room 713, Engineering Building III 1001

Ta-Hsueh Road, Hsinchu, Taiwan

e-mail: ftchien@mail.nctu.edu.tw

\section{R. Y. Chang}

Research Center for Information Technology Innovation, Academia Sinica, 128, Academia Road, Section 2 Nankang,

Taipei 11529, Taiwan

e-mail: yjrchang@gmail.com

\section{M.-K. Chang}

Graduate Institute of Communication Engineering, National Chung Hsing University, 250, Kuo-Kuang Road,

South District, Taichung, Taiwan

e-mail: minkuanc@dragon.nchu.edu.tw

\section{M.-K. Chang}

Department of Electrical Engineering, National Chung Hsing University, 250, Kuo-Kuang Road, South District, Taichung, Taiwan solution concept of the Shapley value to fairly divide the payoffs among the users. Due to the convexity of the proposed game, the Shapley value is shown to be in the core. We derive the optimal strategy for the SU, i.e., transmitting its own data or serving as a relay, that maximizes the sum rate of all PUs and SUs. The payoff allocations according to the core and the Shapley value are illustrated by an example, which demonstrates the benefits of forming the grand coalition as compared with noncoalition and other coalition schemes.

Keywords Coalitional game - Core - Shapley value · Cooperative cognitive radio networks

\section{Introduction}

In recent years, the scarcity of spectral resources has become a severe problem due to the significant growth in commercial wireless services. The traditional fixed spectrum allocation is proved inefficient, since the frequency bands are largely under-utilized [1]. Cognitive Radio (CR) [2] has been considered as a promising technology to improve spectrum utilization by allowing secondary users (SUs) to access spectrum holes unoccupied by primary users (PUs). Recently, with the emergence of cooperative communications in wireless networks [3], a new communication paradigm in cognitive radio networks was proposed [4-6], termed cooperative cognitive radio networks (CCRN). In CCRN, PUs actively select SUs with good channel conditions as their cooperative relays and lease available spectrum to them. By relaying data for PUs, SUs gain opportunities to access the spectrum otherwise unavailable to them. Therefore, a "win-win" situation is created where PUs can potentially increase their 
transmission rates and SUs can access the available frequency bands to transmit their own traffic.

In the conventional CCRN formulation, some type of resource allocation problem was addressed, such as subchannel assignment for SUs, relay assignment, and power control [4-6]. In [4], the subcarrier assignment, relay assignment, and SU relay strategy optimization problems were approached with flexible channel cooperation in a multi-channel CCRN, where a unified optimization framework based on Nash Bargaining Solutions was developed. In $[5,6]$, the spectrum leasing problem was formulated for one PU and multiple SUs as a Stackelberg game and the Nash equilibrium was derived. A single channel was assumed available, and different transmissions were divided in time. The consideration of one channel and one PU in $[5,6]$ presents a simplification for practical scenarios where there are typically multiple channels and multiple PUs that coexist in the coverage area of a base station in the cellular network.

In this paper, we investigate the cooperation strategies between multiple PUs and multiple SUs in a multi-channel CCRN. We propose a coalition game theoretic framework [7-9] to understand how to exploit SUs' involvement so that optimal fraction of time allocation can be obtained. In addition, flexible and effective cooperation among PUs and SUs can be achieved and conflicting interests between them can be resolved. We formulate the problem as a coalition game with transferable utility (TU) $[9,10]$, and analyze the cooperation strategies among PUs and SUs in a multi-channel CCRN. We show that all PUs and SUs will form a grand coalition, and the solution concept, the core [11], is nonempty. Besides, a payoff allocation in the core can be obtained by solving the dual problem of a convex optimization problem $[12,13]$. The main contributions of this paper are summarized as follows.

1. We propose a new coalitional game-theoretic framework to analyze the cooperation behavior between multiple PUs and SUs in a multi-channel CCRN. We apply the solution concepts of the core and the Shapley value, respectively, to characterize the stability and fair allocation of the aggregate utility among rational users.

2. We demonstrate that the formulated coalitional game is a convex game and, as a result, the Shapley value always lies in the core according to [14].

3. We develop a method to determine the optimal relay strategies for SUs based on the channel conditions of the subchannels leased for SUs.

4. We present numerical analyses to verify the benefits of forming a grand coalition among PUs and SUs. An example is also given to illustrate payoff allocations according to both the Shapley value and the core.

The rest of the paper is organized as follows. Section 2 presents the system model and problem formulation. In
Sect. 3, the proposed coalitional game approach is described. Simulation results are given in Sect. 4 Finally, the concluding remark is given in Sect. 5 .

\section{System model}

\subsection{System setup}

We consider the uplink transmission of a single-cell cognitive radio network coexisted with a primary licensed network. The entire network consists of $N_{p}$ PUs, a primary receiver called the primary base station (PBS), $N_{s}$ SUs, and a cognitive receiver called the secondary access point (SAP). Let the set of PUs and SUs be $\mathcal{N}_{p}=\left\{1, \ldots, N_{p}\right\}$ and $\mathcal{N}_{s}=\left\{1, \ldots, N_{s}\right\}$, respectively. Without loss of significant generality we assume that the $i$ th PU communicates with PBS using the $i$ th subchannel only, and the set of all PUs' subchannels in the primary network is $\mathcal{S}_{b}=$ $\left\{1, \ldots, N_{p}\right\}$. The unlicensed cognitive SUs can only transmit their own traffic using the subchannels leased from a group of PUs who are willing to cooperate. The cooperating SUs and PUs form a coalition.

Specifically, a coalition $\mathcal{S}=\mathcal{S}_{p} \cup \mathcal{S}_{s}$ is a subset of $\mathcal{N}_{p} \cup$ $\mathcal{N}_{s}$ in which multiple PUs and SUs cooperate to realize their own benefits, where $\mathcal{S}_{p} \subseteq \mathcal{N}_{p}$ and $\mathcal{S}_{s} \subseteq \mathcal{N}_{s}$. We assume $\mathcal{S}_{p}$ and $\mathcal{S}_{s}$ are not empty in order for the coalition between PUs and SUs to be meaningful. The coalition $\mathcal{N}_{p} \cup \mathcal{N}_{s}$ that includes all PUs and SUs is called the grand coalition. Fig. 1 depicts a simple example of our proposed model with two PUs and two SUs. As depicted, PU 1 and SU 1 form a coalition $\mathcal{S}_{1}$ and PU 2 and SU 2 form another coalition $\mathcal{S}_{2}$, or all of them form a grand coalition. By forming the grand coalition all PUs and SUs may gain additional benefits as summarized as follows:

- For PUs: PUs can select different SUs as relay nodes and can gain more revenues from SUs. For instance, PU 1 can choose either SU 1 or SU 2 as its relay node.

- For SUs: SUs can gain more opportunities to access subchannels leased by different PUs. For instance, SU 1 can access the subchannels leased by PU 1 and PU 2 to increase its own data rates.

The main objective of the work is to justify the above two statements using the tools established in the coalitional game theory [9].

\subsection{Transmission protocol with coalition}

Next, we describe the CCRN cooperation mechanism and assumptions in the following: 
(a)

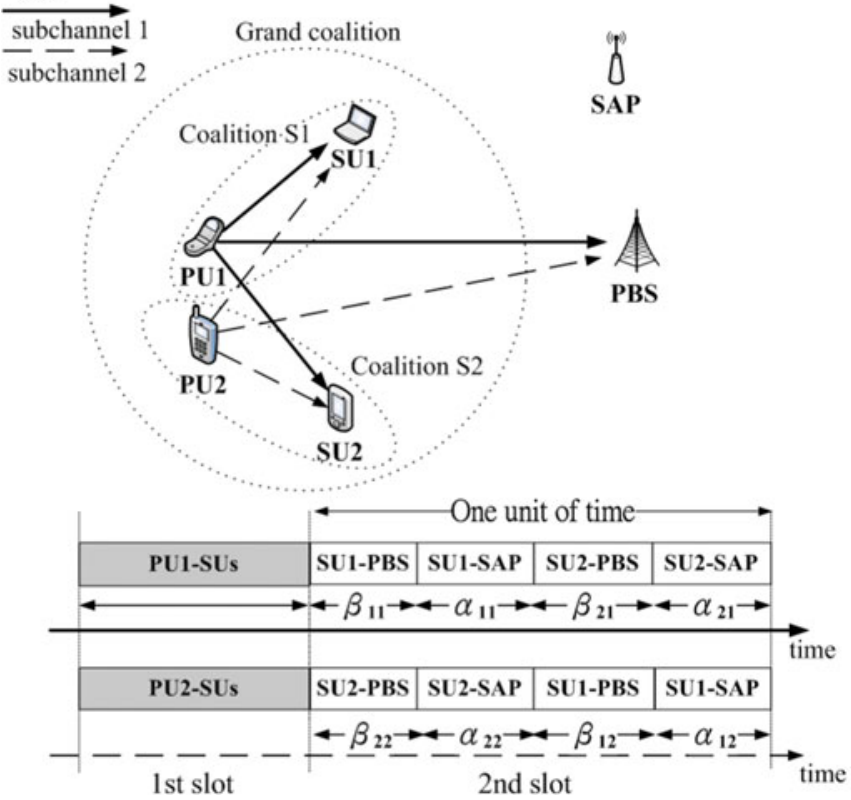

(b)

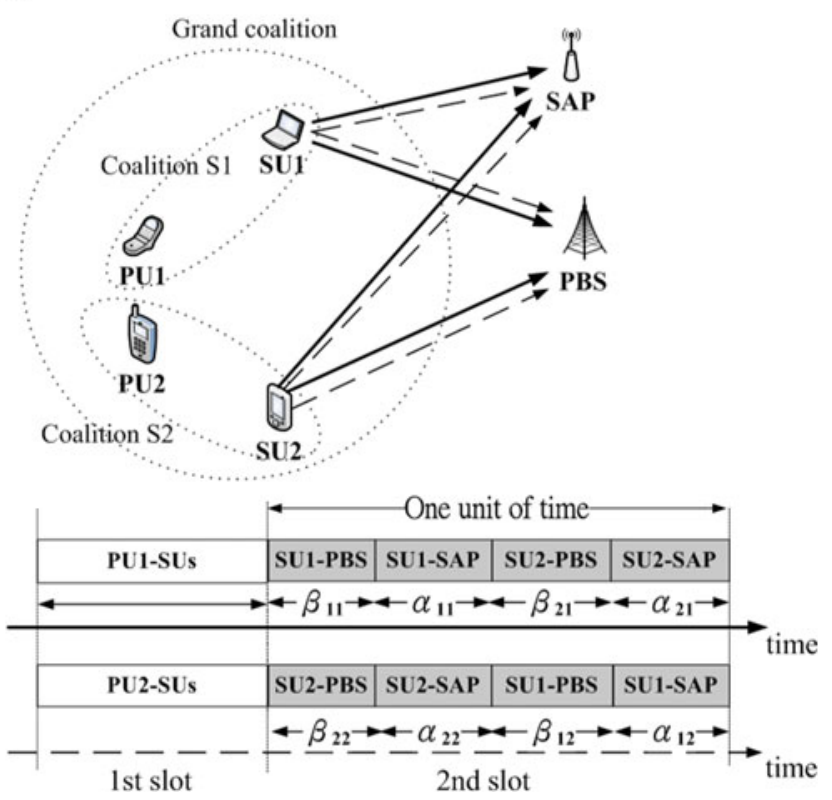

Fig. 1 The proposed network model and its transmission protocol. a In the first time slot, PUs transmit their data to SUs and the PBS. b In the second time slot, SUs relay PUs' traffic to PBS or transmit their own data to SAP

1. SUs are assigned with different subchannels at any given time, i.e., no interference between SUs' transmissions.

2. Each PU has only one available subchannel for lease in the formed coalition. An SU exclusively uses the subchannel $i$ leased by PU $i$ when it relays PU $i$ 's traffic. For example, when SU 2 relays the traffic of PU 1 it always uses subchannel 1. Furthermore, one SU can assist the transmission of at most one PU at a given time.

3. The cooperative transmissions among PUs and SUs are scheduled in a time-division fashion. As shown in Fig. 1, the first time slot is for PUs' transmission and the second time slot for SUs' transmission. The second time slot is further divided into two parts, i.e., SUs relaying PUs' data and transmitting their own data. In the second time slot, the fraction of time that SU $k$ relays PU $m$ 's traffic (using subchannel $m$ ) and transmits its own traffic using subchannel $j$ are denoted by $\beta_{k, m}$ and $\alpha_{k, j}$, respectively, where $0 \leq \beta_{k, m} \leq 1$ and $0 \leq \alpha_{k, j} \leq 1$ for $k \in \mathcal{S}_{s}, m \in \mathcal{S}_{p}, j \in \mathcal{S}_{b}$. The time fractions are computed at the SAP.

4. Amplify-and-forward (AF) protocol is adopted at SUs to relay PUs' traffic, and equal power allocation is employed for all PUs and SUs. Flat Rayleigh fading channel coefficients for each link are assumed invariant within two time slots. At the PBS, the receiver employs maximal ratio combining (MRC) to combine signals from the direct link and the relay links. The channel state information (CSI) is assumed available at the PBS.
5. PUs and SUs are rational entities interested in maximizing their own utility. In addition, PUs and SUs are rightful entities who will honestly report their fading coefficients of their own links (Interesting readers can refer to [21-23] for the issue of cheating behavior in games).

We use the following notations to denote the channel gain between different transmitter-receiver pairs: $h_{p_{m}, P B S}$ denotes the channel gain between PU $m$ and the PBS; $h_{p_{m}, s_{k}}$ denotes the channel gain between PU $m$ and SU $k ; h_{s_{k}}^{(j)}$ denotes the channel gain of subchannel $j$ from SU $k$ to the SAP; and $h_{s_{k}, P B S}^{(j)}$ denotes the channel gain of subchannel $j$ from SU $k$ to the PBS.

\subsection{Utility definition}

With the cooperation mechanism described in the previous section, we are now ready to define each player's utility based on their respective achievable transmission rate. First, the achievable data rate (per Hertz) for PU $m$ with the help of SU $k$ is given by

$R_{m, k, P B S}=\beta_{k, m} \cdot I_{k, m}$,

where

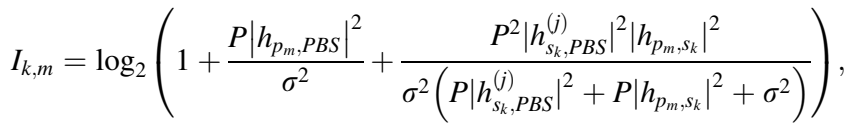


with $P$ being the transmission power and $\sigma^{2}$ the power of the noise. Note that, for notational simplicity, we have assumed the noise powers observed by all receivers are identical. Thus, the transmission rate of PU $m$ is given by

$r_{p_{m}}=\sum_{k \in \mathcal{S}_{s}} R_{m, k, P B S}$

Next, when the subchannel of PU $m$ is leased to SUs, we model the cost of PU $m$ 's spectrum leasing as the total fraction of time that its subchannel is used by SUs, i.e.,

$\varphi_{j}=\sum_{k \in \mathcal{S}_{s}} \alpha_{k, j}$.

Then, the utility of PU $m$ is defined as

$U_{p_{m}}=F\left(r_{p_{m}}\right)+c_{p_{j}}-G\left(\varphi_{j}\right)$,

where $F(\cdot)$ is assumed to be a concave increasing function mapping each PU's rate to a utility gain. For example, utility functions can be linear, that is, $F\left(r_{p_{m}}\right)=r_{p_{m}}$. PU $m$ incurs cost when its subchannel is leased to SUs. We define the cost as a function $G(\cdot)$ of the fraction of time allocation $\alpha_{k, j}$, where $G(\cdot)$ is assumed to be an increasing convex function. Besides, $c_{p_{j}}=\omega_{s} \cdot \sum_{k \in \mathcal{S}_{s}} \alpha_{k, j}$ is the payment of SUs to PU $m$ with $\omega_{s}$ representing the price of spectrum use per unit time.

On the other hand, the SUs as cognitive relays have opportunities to use the spare subchannels to transmit their traffic. The data rate of SU $k$ is

$r_{s_{k}}=\sum_{j \in \mathcal{S}_{b}} R_{k}^{(j)}$,

where $R_{k}^{(j)}=\alpha_{k, j} \cdot R_{k, j}$ is the maximum achievable data rate of SU $k$ using subchannel $j$ to transmit its traffic to SAP, and $R_{k, j}=\log _{2}\left(1+\frac{P\left|h_{s^{\prime}}\right|^{2}}{\sigma^{2}}\right)$. Then, the utility of SU $k$ is defined as

$U_{s_{k}}=H\left(r_{s_{k}}\right)-c_{s_{k}}$,

where $H(\cdot)$ is assumed to be an increasing concave function which projects rate to revenue, and could be a linear function. In addition, $c_{s_{k}}=\omega_{s} \cdot \sum_{j \in \mathcal{S}_{b}} \alpha_{k, j}$ which represents the SU k's payment to PUs.

\section{Game theoretic formulation and analysis}

A coalitional game can be classified as either a transferable utility (TU) game or a non-transferable utility (NTU) game [9]. A TU game is one in which the yield of a coalition (i.e., the coalition value) can be apportioned arbitrarily between the coalition members, and an NTU game is one in which this apportioning depends on the joint actions of the members in the coalition. As will be seen shortly, since the coalition value can be properly defined as the sum of utility generated by PUs and SUs in the coalition, a TU game applies to our considered network. In addition, as will be shown in this section, our considered network satisfies the properties of a canonical coalitional game, e.g., grand coalition. Therefore, we formulate our considered cognitive radio network as a canonical coalitional game with TU. In the following, we first present some coalitional game-related terminologies and then show that this coalitional game has desired properties that ensure a stable grand coalition and a fair payoff allocation.

A coalitional game with TU is uniquely defined by the set of players who seek to form cooperative groups and the coalition value which associates each nonempty subset of players with a real number [9]. First, we give the definition of the coalition value for our considered game.

Definition 1 In the considered canonical coalitional game with $\mathrm{TU}$, the coalition value $v(\mathcal{S})$ of a coalition $\mathcal{S}$ is a real number defined as the maximum sum utility generated by PUs and SUs in $\mathcal{S}$, which depends only on the actions of the PUs and SUs in $\mathcal{S}$ and not those in $\mathcal{N}_{p} \cup \mathcal{N}_{s} \backslash \mathcal{S}$. By Definition 1 and using the utility definitions in (5) and (7), the coalition value $v(\mathcal{S})$ is the solution to the following convex optimization problem:

$\mathbf{P}(\mathcal{S}): v(\mathcal{S}) \triangleq \max _{\boldsymbol{\alpha}, \boldsymbol{\beta}} \sum_{m \in \mathcal{S}_{p}} U_{p_{m}}+\sum_{k \in \mathcal{S}_{s}} U_{s_{k}}$

subject to $\sum_{j \in \mathcal{S}_{b}} \alpha_{k, j}+\sum_{m \in \mathcal{S}_{p}} \beta_{k, m} \leq 1, k \in \mathcal{S}_{s}$,

$\sum_{k \in \mathcal{S}_{s}}\left(\alpha_{k, j}+\beta_{k, m}\right) \leq 1, j \in \mathcal{S}_{b}, m \in \mathcal{S}_{p}$,

$0 \leq \alpha_{k, j} \leq 1,0 \leq \beta_{k, m} \leq 1$,

where $U_{p_{-} m}$ and $U_{s_{-} k}$ are given in (5) and (7), respectively, and the set of time fractions we need to solve in (8) are $\boldsymbol{\alpha}=\left\{\alpha_{k, j}: k \in \mathcal{S}_{s}, j \in \mathcal{S}_{b}\right\}$ and $\boldsymbol{\beta}=\left\{\beta_{k, m}: k \in \mathcal{S}_{s}, \quad m \in\right.$ $\left.\mathcal{S}_{p}\right\}$. Note that $\boldsymbol{\alpha}$ is in $U_{p_{m}}$ and $U_{s_{k}}$, while $\boldsymbol{\beta}$ resides in $U_{p_{m}}$. The constraint in (9) guarantees that each SU works in either relay or access mode but not simultaneously, and (10) states that the total amount of spectrum-accessing time fraction for an $\mathrm{SU}$ in coalition $\mathcal{S}$ cannot exceed one. The constraint in (11) guarantees a feasible joint action of coalition $\mathcal{S}$ [12]. If the solution to (8) is not feasible, then $v(\mathcal{S}) \triangleq-\infty$.

\subsection{The core}

As mentioned previously, for a TU game, the coalition value can be apportioned or shared in any manner among 
the cooperating PUs and SUs. A specific sharing strategy is called a payoff allocation strategy. A feasible payoff allocation strategy for a canonical coalitional game is one that ensures if a subset of players separates from the grand coalition, at least one PU or SU in the separated subset has a utility worse than that in the grand coalition. To obtain a feasible payoff allocation strategy, we use the core solution concept [9], which is formally defined as follows.

Definition 2 The core is the set of feasible payoff allocation vectors $\mathbf{x}=\left[x_{p_{1}}, \ldots, x_{p_{N_{p}}}, x_{s_{1}}, \ldots, x_{s_{N_{s}}}\right]$ for the grand coalition, which is said to be an imputation if $\sum_{m \in \mathcal{N}_{p}} x_{p_{m}}+$ $\sum_{k \in \mathcal{N}_{s}} x_{s_{k}}=v\left(\mathcal{N}_{p} \cup \mathcal{N}_{s}\right)$ and $x_{i} \geq v(\{i\}), \forall i \in\left(\mathcal{N}_{p} \cup \mathcal{N}_{s}\right)$, where $x_{p_{m}}$ and $x_{s_{k}}$ represent the payoff value of PU $m$ and $\mathrm{SU} k$, respectively. The core $\mathcal{C}$ is the set of imputations for which $\sum_{m \in \mathcal{S}_{p}} x_{p_{m}}+\sum_{k \in \mathcal{S}_{s}} x_{s_{k}} \geq v(\mathcal{S})$ for all $\mathcal{S} \subset \mathcal{N}_{p} \cup \mathcal{N}_{s}$, i.e.,

$$
\begin{aligned}
\mathcal{C}= & \left\{\mathbf{x}: \sum_{m \in \mathcal{N}_{p}} x_{p_{m}}+\sum_{k \in \mathcal{N}_{s}} x_{s_{k}}=v\left(\mathcal{N}_{p} \cup \mathcal{N}_{s}\right)\right. \text { and } \\
& \left.\sum_{m \in \mathcal{S}_{p}} x_{p_{m}}+\sum_{k \in \mathcal{S}_{s}} x_{s_{k}} \geq v(\mathcal{S}) \quad \forall \mathcal{S} \subset \mathcal{N}_{p} \cup \mathcal{N}_{s}\right\} .
\end{aligned}
$$

Definition 2 states that, the core, if exists, provides a set of stable payoff allocations because no coalition $\mathcal{S} \subset$ $\mathcal{N}_{p} \cup \mathcal{N}_{s}$ has the incentives to reject the proposed payoff allocation and leave the grand coalition to form a separate coalition instead (having a stable grand coalition).

To show that our proposed game has a nonempty core and obtain the payoff allocation for PUs and SUs, first, we employ the duality theorem [13] to transform the primal problem in (8) into its dual problem. By the transformation, the relation of the solution set between the dual problem and its respective primal problem can be obtained; that is, the optimal solution to the dual problem is the upper bound of the optimal solution to the primal problem [15]. Besides, through the transformation, the solution set of the dual problem can be shown to be a subset of the core, as will be stated by Theorem 1 . First, the Lagrangian functions of PUs and SUs are defined as

$$
\begin{aligned}
f_{m, j}(\delta, \theta, \pi)= & \max _{r_{p_{m}} \geq 0, \varphi_{j} \geq 0, c_{p_{j}} \geq 0, m \in \mathcal{S}_{p}, j \in \mathcal{S}_{b}}\left(F\left(r_{p_{m}}\right)+c_{p_{j}}\right. \\
& \left.-G\left(\varphi_{j}\right)+\delta_{m} r_{p_{m}}+\theta_{j} c_{p_{j}}+\pi_{j} \varphi_{j}\right) \\
g_{k}(\gamma, \tau)= & \max _{c_{s_{k}} \geq 0, r_{s_{k}} \geq 0, k \in \mathcal{S}_{s}}\left(H\left(r_{s_{k}}\right)-c_{s_{k}}+\gamma_{k} r_{s_{k}}+\tau_{k} c_{s_{k}}\right)
\end{aligned}
$$

where $\delta_{m}, \theta_{j}, \pi_{j}, \gamma_{k}$ and $\tau_{k}$ are the Lagrange multipliers. Then, the dual problem of the problem in (8) for $\mathcal{S}=$ $\mathcal{S}_{p} \cup \mathcal{S}_{s}$ is formulated as

$$
\begin{gathered}
\mathbf{D}(\mathcal{S}): \min _{\boldsymbol{\alpha}, \boldsymbol{\beta}} \sum_{m \in \mathcal{S}_{p}} \sum_{j \in \mathcal{S}_{b}}\left(f_{m, j}(\delta, \theta, \pi)+\eta_{m, j}\right) \\
+\sum_{k \in \mathcal{S}_{s}}\left(g_{k}(\gamma, \tau)+\lambda_{k}\right)
\end{gathered}
$$

subject to $\theta_{j}+\gamma_{k} R_{k, j}+\omega_{s} \pi_{j}+\omega_{s} \tau_{k}+\sum_{m \in \mathcal{S}_{p}} \eta_{m, j} \geq 0$,

$$
\begin{aligned}
& k \in \mathcal{S}_{s}, j \in \mathcal{S}_{b}, \\
& \delta_{m} I_{k, m}+\lambda_{k}+\sum_{j \in \mathcal{S}_{b}} \eta_{m, j} \geq 0, k \in \mathcal{S}_{s}, m \in \mathcal{S}_{p}, \\
& \tau_{k}, \lambda_{k}, \gamma_{k} \geq 0, k \in \mathcal{S}_{s}, \\
& \theta_{j}, \pi_{j} \geq 0, j \in \mathcal{S}_{b} \\
& \eta_{m, j} \geq 0, m \in \mathcal{S}_{p}, j \in \mathcal{S}_{b}, \\
& \delta_{m} \geq 0, m \in \mathcal{S}_{p}
\end{aligned}
$$

where $\eta_{m, j}$ and $\lambda_{k}$ are also the Lagrange multipliers which are derived from the transformation of the primal problem in (8) into its dual problem in (12). Let $\mathcal{D}$ constitute the set of optimal solutions of the above optimization problem for $\mathcal{S}_{p}=\mathcal{N}_{p}$ and $\mathcal{S}_{s}=\mathcal{N}_{s}$, and let

$$
\begin{gathered}
\mathcal{O}=\left\{\mathbf{x}^{*}: x_{p_{m}}^{*}=f_{m, j}\left(\delta^{*}, \theta^{*}, \pi^{*}\right)+\eta_{m, j}^{*}, m \in \mathcal{N}_{p}, j \in \mathcal{S}_{b},\right. \\
x_{s_{k}}^{*}=g_{k}\left(\gamma^{*}, \tau^{*}\right)+\lambda_{k}^{*}, k \in \mathcal{N}_{s}, \\
\text { for some } \left.\left(\delta^{*}, \theta^{*}, \pi^{*}, \eta^{*}, \gamma^{*}, \tau^{*}, \lambda^{*}\right) \in \mathcal{D}\right\}
\end{gathered}
$$

represent the dual payoff generated by the solution of the dual problem. The dual payoff is the set of PU's and SU's optimal payoff allocation. Then, we show the core of the proposed game is nonempty in the following theorem.

Theorem 1 The core of the considered canonical coalitional game with $T U$ is nonempty and $\mathcal{O} \subseteq \mathcal{C}$.

Proof Since the set $\mathcal{D}$ is nonempty, the set $\mathcal{O}$ is also nonempty. An arbitrary $\mathbf{x}^{*} \in \mathcal{O}$ corresponding to some $\left(\delta^{*}, \theta^{*}, \pi^{*}, \eta^{*}, \gamma^{*}, \tau^{*}, \lambda^{*}\right) \in \mathcal{D}$ is considered. Now, the value $\sum_{m \in \mathcal{S}_{p}} x_{p_{m}}^{*}+\sum_{k \in \mathcal{S}_{s}} x_{S_{k}}^{*}$ is the optimal value of the objective function of $\mathbf{D}\left(\mathcal{N}_{p} \cup \mathcal{N}_{s}\right)$. Since $F(\cdot)$ and $H(\cdot)$ are concave, and $G(\cdot)$ is convex, the objective function of (8) is concave. In addition, we see the constraints (9)-(11) are linear. Since $\mathbf{D}(\mathcal{S})$ is the dual of the optimization problem in (8) for each $\mathcal{S} \subset \mathcal{N}_{p} \cup \mathcal{N}_{s}$, by the definition of strong duality [13], the duality gap is zero. Thus, $\sum_{m \in \mathcal{S}_{p}} x_{p_{m}}^{*}+\sum_{k \in \mathcal{S}_{s}} x_{s_{k}}^{*}=$ $v\left(\mathcal{N}_{p} \cup \mathcal{N}_{s}\right)$. We only need to show that $v(\mathcal{S}) \leq$ $\sum_{m \in \mathcal{S}_{p}} x_{p_{m}}^{*}+\sum_{k \in \mathcal{S}_{s}} x_{s_{k}}^{*}$ for all $\mathcal{S} \subset \mathcal{N}_{p} \cup \mathcal{N}_{s}$.

Let $(8)$ be feasible. Then, by strong duality, $v(\mathcal{S})$ equals the optimal value of the objective function of $\mathbf{D}(\mathcal{S})$. The sub-vectors $(\delta, \theta, \pi, \eta, \gamma, \tau, \lambda)$ consisting of the components of $\left(\delta^{*}, \theta^{*}, \pi^{*}, \eta^{*}, \gamma^{*}, \tau^{*}, \lambda^{*}\right)$ in $\mathcal{S}$ satisfy the constraints of $\mathbf{D}(\mathcal{S})$. Thus, $\sum_{m \in \mathcal{S}_{p}} x_{p_{m}}^{*}+\sum_{k \in \mathcal{S}_{s}} x_{s_{k}}^{*}$ is the value of the objective function of $\mathbf{D}(\mathcal{S})$ for the above feasible 
solution. It follows that the optimal value of the objective function of $\mathbf{D}(\mathcal{S})$ is a lower bound for $\sum_{m \in \mathcal{S}_{p}} x_{p_{m}}^{*}+$ $\sum_{k \in \mathcal{S}_{s}} x_{s_{k}}^{*}$. Thus, $\mathbf{x}^{*} \in \mathcal{C}$.

From the above proof, the optimal payoff vector $\mathbf{x}^{*}$ is in the core and can be obtained by solving the dual problem $\mathbf{D}\left(\mathcal{N}_{p} \cup \mathcal{N}_{s}\right)$. In other words, the solution of the dual problem $\mathbf{D}\left(\mathcal{N}_{p} \cup \mathcal{N}_{s}\right)$ constitutes PU's optimal payoff allocation $x_{p_{m}}^{*}$ and SU's optimal payoff allocation $x_{s_{k}}^{*}$ in the core.

\subsection{Shapley value}

The core as a solution concept has some drawbacks [9]: it may be empty, and the resulting payoff allocation may be unfair. The unfairness results from the core not taking into account the contribution of each user in a coalition. To fairly divide the aggregate payoff among the users, we propose to use the Shapley value solution concept $[11,16]$, which is defined as follows.

Definition 3 The Shapley value assigns the payoff allocation vector $\phi(v)=\left(\phi_{1}(v), \ldots, \phi_{N}(v)\right)$, where

$\phi_{i}(v)=\sum_{\mathcal{S} \subseteq \mathcal{N} \backslash\{i\}} \frac{|\mathcal{S}| !(|\mathcal{N}|-|\mathcal{S}|-1) !}{|\mathcal{N}| !}[v(\mathcal{S} \cup\{i\})-v(\mathcal{S})]$

is the payoff allocated to user $i$ with $\mathcal{N}$ being the grand coalition. The difference $v(\mathcal{S} \cup\{i\})-v(\mathcal{S})$ represents the marginal contribution of user $i$ to a set $\mathcal{S} \subseteq \mathcal{N} \backslash\{i\}$, and therefore $\phi_{i}(v)$ yields the average marginal contribution of user $i$ in any coalition $\mathcal{S}$. In our proposed coalitional game, the Shapley value of each PU and SU can be derived from the combination of (8) and (14).

Theorem 2 The proposed game is a convex game.

Proof According to [17, 18], the supermodularity implies the convexity of a game. The proposed game is supermodular [eqn. (1), 19] due to the nonnegativeness of the second derivative of the proposed coalition value function (8) in the system.

Theorem 3 The Shapley value of the proposed coalitional game always lies in the core.

Proof Since the proposed game is shown to be a convex game, the payoff allocation vector $\phi(v)$ of the Shapley value, which apportions the total payoff fairly among all the users, always lies in the core $[14,20]$.

\section{Simulation results}

In this section, numerical results are presented to illustrate the cooperative actions among PUs and SUs and their

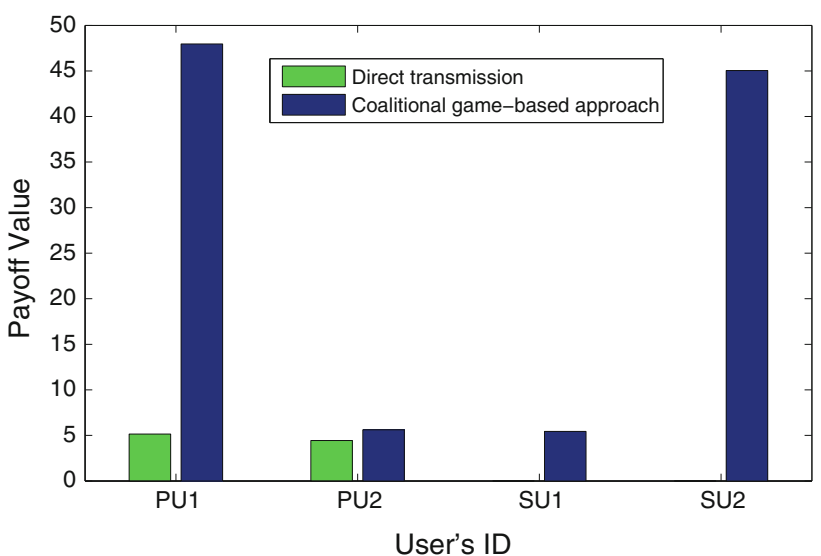

Fig. 2 Comparison of PUs' and SUs' payoffs between the proposed cooperative approach and the direct transmission approach
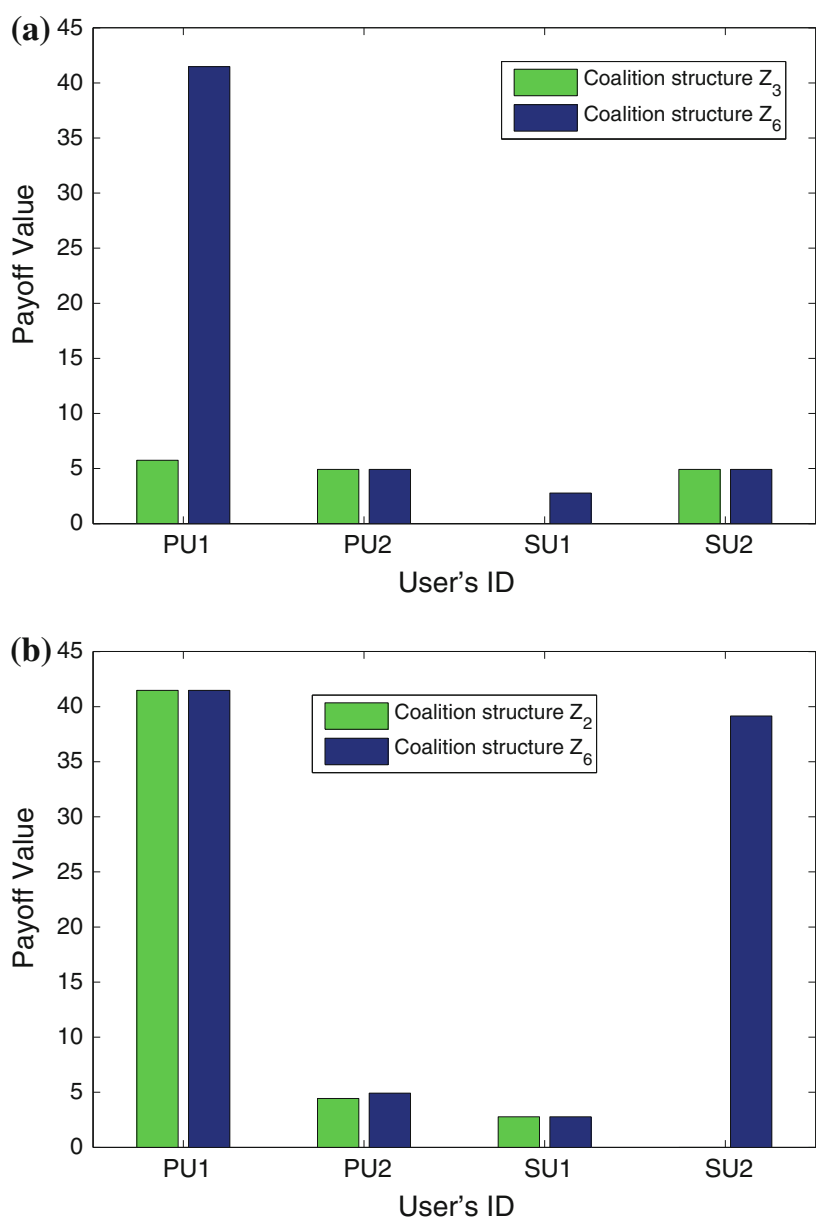

Fig. 3 The benefits of forming coalitions between a PU 1 and SU 1 in $\mathcal{Z}_{6}$ versus noncooperation in $\mathcal{Z}_{3}$, b PU 2 and SU 2 in $\mathcal{Z}_{6}$ versus noncooperation in $\mathcal{Z}_{2}$.

individual benefits gained from cooperation in our considered network. We simulate a scenario where there are two PUs (PU 1 and PU 2) and two SUs (SU 1 and SU 2), but the number of subchannel of each PU for lease is 
Table 1 Payoff allocation for the considered coalitional game

\begin{tabular}{|c|c|c|c|c|c|}
\hline Coalition structure & PU 1 & PU 2 & SU 1 & SU 2 & $v\left(\mathcal{Z}_{i}\right)$ \\
\hline \multicolumn{6}{|l|}{ The dual payoff profile in the core } \\
\hline $\mathcal{Z}_{1}=\{\{P U 1\},\{P U 2\},\{S U 1\},\{S U 2\}\}$ & 5.750 & 4.440 & 0 & 0 & 10.190 \\
\hline $\mathcal{Z}_{2}=\{\{P U 1, S U 1\},\{P U 2\},\{S U 2\}\}$ & 41.480 & 4.440 & 2.780 & 0 & 48.700 \\
\hline $\mathcal{Z}_{3}=\{\{P U 2, S U 2\},\{P U 1\},\{S U 1\}\}$ & 5.750 & 4.920 & 0 & 39.150 & 49.820 \\
\hline $\mathcal{Z}_{4}=\{\{P U 1, S U 2\},\{P U 2\},\{S U 1\}\}$ & 43.410 & 4.440 & 0 & 13.550 & 61.400 \\
\hline $\mathcal{Z}_{5}=\{\{P U 2, S U 1\},\{P U 1\},\{S U 2\}\}$ & 5.750 & 13.910 & 41.220 & 0 & 60.880 \\
\hline $\mathcal{Z}_{6}=\{\{P U 1, S U 1\},\{P U 2, S U 2\}\}$ & 41.480 & 4.920 & 2.780 & 39.150 & 88.380 \\
\hline $\mathcal{Z}_{7}=\{\{P U 1, S U 2\},\{P U 2, S U 1\}\}$ & 12.041 & 27.743 & 6.234 & 39.149 & 85.171 \\
\hline $\mathcal{Z}_{8}=\{\{P U 1, S U 1, P U 2\},\{S U 2\}\}$ & 41.880 & 4.900 & 0.930 & 0 & 47.709 \\
\hline $\mathcal{Z}_{9}=\{\{P U 1, S U 2, P U 2\},\{S U 1\}\}$ & 43.810 & 1.910 & 0 & 5.590 & 51.311 \\
\hline $\mathcal{Z}_{10}=\{\{P U 1, P U 2, S U 1, S U 2\}\}$ & 47.960 & 5.610 & 5.440 & 45.040 & 104.050 \\
\hline \multicolumn{6}{|l|}{ The shapley value } \\
\hline $\mathcal{Z}_{1}=\{\{P U 1\},\{P U 2\},\{S U 1\},\{S U 2\}\}$ & 5.750 & 4.440 & 0 & 0 & 10.190 \\
\hline $\mathcal{Z}_{2}=\{\{P U 1, S U 1\},\{P U 2\},\{S U 2\}\}$ & 25.005 & 4.440 & 19.255 & 0 & 48.700 \\
\hline $\mathcal{Z}_{3}=\{\{P U 2, S U 2\},\{P U 1\},\{S U 1\}\}$ & 5.750 & 24.255 & 0 & 19.815 & 49.820 \\
\hline $\mathcal{Z}_{4}=\{\{P U 1, S U 2\},\{P U 2\},\{S U 1\}\}$ & 31.355 & 4.440 & 0 & 25.605 & 61.400 \\
\hline $\mathcal{Z}_{5}=\{\{P U 2, S U 1\},\{P U 1\},\{S U 2\}\}$ & 5.750 & 29.785 & 25.345 & 0 & 60.880 \\
\hline $\mathcal{Z}_{6}=\{\{P U 1, S U 1\},\{P U 2, S U 2\}\}$ & 25.005 & 24.255 & 19.255 & 19.815 & 88.380 \\
\hline $\mathcal{Z}_{7}=\{\{P U 1, S U 2\},\{P U 2, S U 1\}\}$ & 28.465 & 19.215 & 14.776 & 22.715 & 85.171 \\
\hline $\mathcal{Z}_{8}=\{\{P U 1, S U 1, P U 2\},\{S U 2\}\}$ & 7.778 & 12.558 & 27.373 & 0 & 47.709 \\
\hline $\mathcal{Z}_{9}=\{\{P U 1, P U 2, S U 2\},\{S U 1\}\}$ & 14.782 & 7.682 & 0 & 28.847 & 51.311 \\
\hline $\mathcal{Z}_{10}=\{\{P U 1, P U 2, S U 1, S U 2\}\}$ & 32.005 & 19.022 & 25.775 & 27.248 & 104.050 \\
\hline
\end{tabular}

assumed to be one for simplifying the protocol designs. All PUs and SUs are randomly placed in the same coverage area. The channel gains of link pairs among PUs, SUs, the PBS, and the SAP are independently generated from complex Gaussian distribution with zero mean and unit variance. From Theorem 2, since our proposed game is a convex game, the core of the proposed game always exists for any set of channel realizations of links. Thus, an arbitrary set of channel gains of links among PUs, SUs, the PBS, and the SAP is selected to demonstrate the capability of this work. The channel realizations used to show our simulation results are summarized as follows:

1. PUs-PBS links: The channel gains of PU 1-PBS and PU 2-PBS links are 0.06 and 0.07 , respectively.

2. PUs-SUs links: The channel gains of PU 1-SU 1 and PU 1-SU 2 links are 1.14 and 1.26, respectively; the channel gains of PU 2-SU 1 and PU 2-SU 2 links are 0.87 and 0.33 , respectively.

3. SUs-PBS links: The channel gains of SU 1-PBS link using subchannel 1 and subchannel 2 are 1.11 and 0.77, respectively; the channel gains of SU 2-PBS link using subchannel 1 and subchannel 2 are 0.76 and 0.21 , respectively.

4. SUs-SAP links: The channel gains of SU 1-SAP link using subchannel 1 and subchannel 2 are 0.13 and
0.56, respectively; the channel gains of SU 2-SAP link using subchannel 1 and subchannel 2 are 0.91 and 0.82 , respectively.

We assume that the noise power at all receivers is $\sigma^{2}=10^{-2}$, and the transmission power for all PUs and SUs is $P=1$. The number of available subchannels is chosen to be one for both PU 1 and PU 2. The price of spectrum use per unit time is set to $\omega_{s}=5$. Linear utility functions are adopted, i.e., $F\left(r_{p_{m}}\right)=10 \times r_{p_{m}}, G\left(\varphi_{m}\right)=$ $7 \times \varphi_{m}$, and $H\left(r_{s_{k}}\right)=g_{a} \times r_{s_{k}}$, where $g_{a}$ is the adjustable access data rate gain indicating the data rate gain of SUs through cooperation with PUs.

Figure 2 shows the comparison result of the payoff allocation for PUs and SUs in the core, with the access data rate gain $g_{a}$ set to 10 . Recall that the solution to the dual problem $\mathbf{D}\left(\mathcal{N}_{p} \cup \mathcal{N}_{s}\right)$ determines PUs' and SUs' payoff allocation in the core. As can be clearly seen, the cooperative benefits for all PUs and SUs from forming the grand coalition as compared to the case in which PUs and SUs do not cooperate are significant. For instance, before forming the grand coalition the utility of SU 1 and SU 2 is zero, since they have no spectrum access; after forming the grand coalition, the payoff of SU 1 and SU 2 increase to 5.44 and 45.04, respectively. For PUs, without the cooperation from SUs the payoff of direct transmission of PU 1 


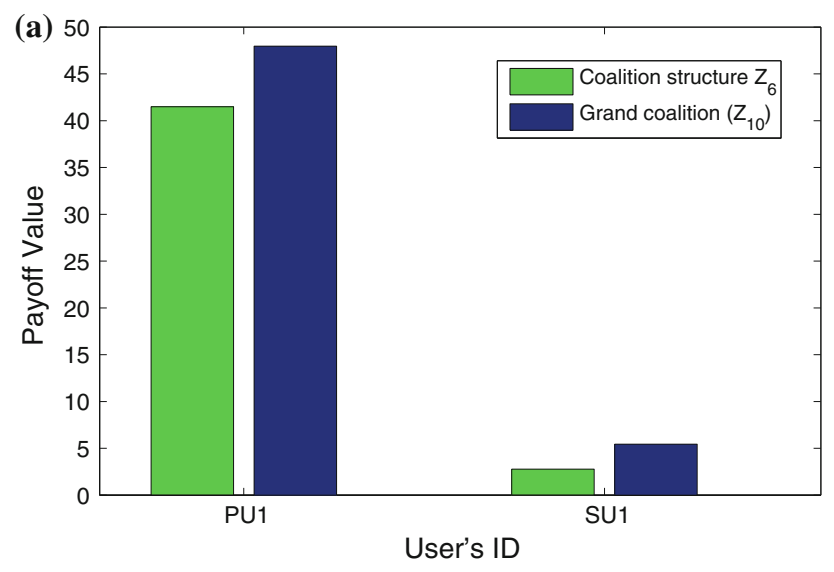

For PU 1 and SU 1.

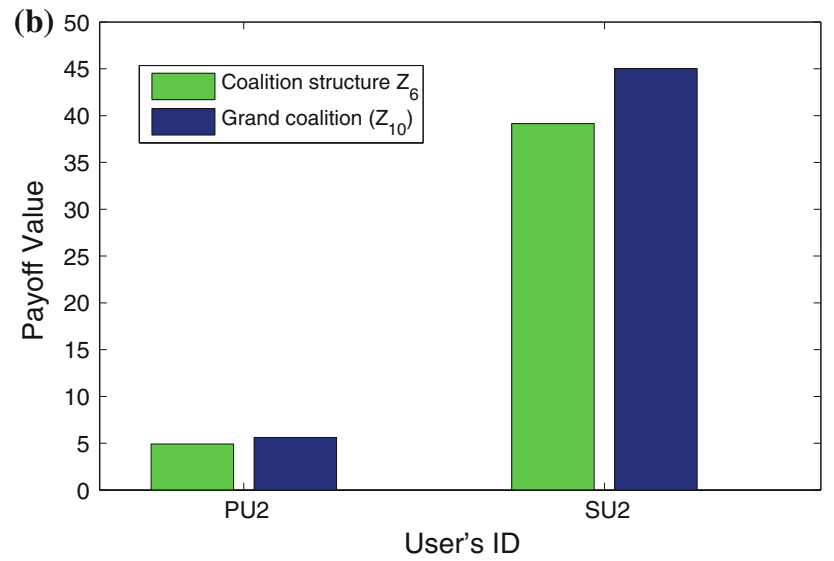

For PU 2 and SU 2.

Fig. 4 Comparison of PUs' and SUs' payoff when forming separate coalitions $\mathcal{S}_{1}$ (PU 1 and SU 1) and $\mathcal{S}_{2}$ (PU 2 and SU 2) versus when forming the grand coalition

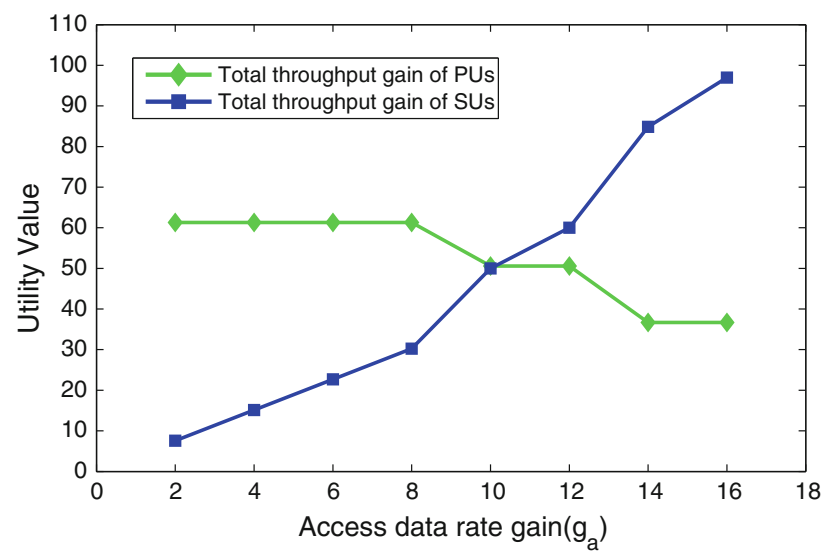

Fig. 5 The impact of access data rate gain $\left(g_{a}\right)$ on the total throughput gain of PUs and SUs in the considered network

and PU 2 are 5.75 and 4.44, respectively; after forming the grand coalition, the payoff increases to 47.96 and 5.61, respectively.
Figure 2 also shows the SUs' ability to select subchannels with better channel conditions in the grand coalition, and adjust the fraction of time for relay or for transmitting its own traffic. For this particular channel realization, since subchannel 1 has superior gain on the SU 1-PBS link, SU 1 uses the majority of time to relay PU 1's traffic using subchannel 1, resulting in the significant increase in PU 1's throughput (from 5.75 to 47.96). Similarly, since subchannel 2 has poorer channel condition on the SU 2-PBS link than on the SU 2-SAP link, SU 2 uses a large portion of time to transmit its own traffic using subchannel 2 to achieve a higher utility. Therefore, both PUs and SUs benefit from the grand coalition.

Figure $3(a, b)$ show the benefits for PUs and SUs of forming the coalitions. The payoff of PU 1 in the structure $\mathcal{Z}_{6}$ of Table 1 increases significantly as compared to the case in which PU 1 and SU 1 do not cooperate in the structure $\mathcal{Z}_{3}$. The payoff of SU 2 in the structure $\mathcal{Z}_{6}$ of Table 1 increases significantly as compared to the case in which PU 2 and SU 2 do not cooperate in the structure $\mathcal{Z}_{2}$.

Figure 4 illustrates the total utility in the network after forming the grand coalition as compared to the coalition structure $\mathcal{Z}_{6}$ of Table 1 where two separate coalitions $\mathcal{S}_{1}$ (PU 1 and SU 1) and $\mathcal{S}_{2}$ (PU 2 and SU 2) are formed. The benefits of forming the grand coalition can be clearly seen, as both PU 1 and SU 1 (PU 2 and SU 2) achieve higher utility evaluations in the grand coalition than in coalition $\mathcal{S}_{1}\left(\mathcal{S}_{2}\right)$. This is because when PUs and SUs form the grand coalition, PUs have more relay options and can gain more revenues from SUs' payment, and SUs have more opportunities to access the spare subchannels. Fig. 4 confirms the superadditivity of the considered coalitional game and a stable grand coalition as stated in Theorem 1.

Figure 5 depicts the impact of the access data rate gain $g_{a}$ on the cooperation scheme. We see that, as $g_{a}$ increases, the SUs' total throughput gain increases while the PUs' total throughput gain declines. This can be explained as follows. With a larger $g_{a}$, increasing $r_{s k}$ will give the objective function in (8) a higher total value, thereby yielding a higher SUs' total throughput gain in Fig. 5. Also, from (6), increasing $r_{s k}$ means that the value $\alpha_{k, j}$ goes up, which implies a smaller $\beta_{k, m}$ according to the constraint in (10). It follows that the PUs' total throughput gain decreases.

Finally, we demonstrate the fairness characteristic of the Shapley value in Table 1, which shows the results of payoff allocations from the core and the Shapley value solutions of the coalitional game for different coalition structures, denoted by $\mathcal{Z}_{1}-\mathcal{Z}_{10}$. The sum coalition value denoted by $v\left(\mathcal{Z}_{i}\right)$ in the last column of Table 1 is defined as the sum of coalition values of all coalitions in each structure $\mathcal{Z}_{i}, i=1,2, \ldots, 10$. In other words, it is the sum of all users' payoff allocations. Note that, in each possible 
coalition of each coalition structure, the payoff allocation is calculated using (14). It is seen that the grand coalition, i.e., $\mathcal{Z}_{10}$, has the maximum sum coalition value.

It can also be observed that the Shapley value solution fairly divides the payoffs among the users in the system. For instance, in the coalition structure $\mathcal{Z}_{2}$ of Table 1 , due to the coalition formed by PU 1 and SU 1, the marginal contributions of PU $1\left(M C_{P U_{1}}\right)$ and $\mathrm{SU} 1\left(M C_{S U_{1}}\right)$ are respectively given by

$$
\begin{aligned}
& M C_{P U_{1}}=(41.480+2.780)-0=44.260, \\
& M C_{S U_{1}}=(41.480+2.780)-5.750=38.510 .
\end{aligned}
$$

From the above calculations, it can be found that since the marginal contribution of PU 1 is larger than that of SU 1, the payoffs of PU 1 allocated by the dual payoff and the Shapley value are larger than those of SU 1. This shows the obtained users payoffs are directly proportional to their marginal contributions. In addition, from Table 1 , we can find that the differences of the payoffs of users who are in the same coalition allocated by the Shapley value are smaller than those by the dual payoff. For instance, in the coalition structure $\mathcal{Z}_{2}$, the payoffs of PU 1 and SU 1 allocated by the dual payoff are 41.480 and 2.780, respectively, and the payoffs of PU 1 and SU 1 allocated by the Shapley value are 25.005 and 19.255 , respectively. Accordingly, the differences of the allocated payoff between PU 1 and SU 1 in the Shapley value is 5.750 which is significantly smaller than that in the dual payoff, whose value is 38.700 . This means that SU 1 , when cooperating with PU 1 in $\mathcal{Z}_{2}$, is more fairly treated with the Shapley value.

\section{Conclusion}

We have proposed a game-theoretic approach to studying the cooperation strategies between multiple PUs and multiple SUs in a multi-channel CCRN. The proposed spectrum-sharing model takes full advantage of the channel diversity and user diversity, and allows SUs to freely customize their use of leased resources. We investigated the problem of payoff maximization for all PUs and SUs in the network via the established coalitional game model. We used the core and the Shapley value solution concept to characterize the stability and fair allocation of the payoff among users, respectively. The analytical arguments were verified by simulation, where the benefits of forming the grand coalition were visually presented for all PUs and SUs in the multi-channel CCRN. Interesting topics for future work include power control, relay node and subchannel selection, and distributed algorithms for achieving payoff allocation in an extension of the proposed framework.

\section{References}

1. Akyildiz, I. F., Lee, W.-Y., Vuran, M. C., \& Mohanty, S. (2006). Next generation/dynamic spectrum access/cognitive radio wireless networks: A survey. Elseiver Computer Networks, 50(13), $2127-2159$.

2. Haykin, S. (2005). Cognitive radio: Brain-empowered wireless communications. IEEE Journal on Selected Areas in Communications, 23(2), 201-220.

3. Laneman, J., Tse, D., \& Wornell, G. (2004). Cooperative diversity in wireless networks: Efficient protocols and outage behavior. IEEE Transactions on Information Theory, 50(12), 3062-3080.

4. Xu, H., \& Li, B. (2010). Efficient resource allocation with flexible channel cooperation in OFDMA cognitive radio networks. In Proceedings of IEEE INFOCOM 2010, March 2010.

5. Simeone, O., Stanojev, I., Savazzi, S., Bar-Ness, Y., Spagnolini, U., \& Pickholtz, R. (2008). Spectrum leasing to cooperating secondary ad hoc networks. IEEE Journal on Selected Areas in Communications, 26(1), 203-213.

6. Zhang, J., \& Zhang, Q. (2009). Stackelberg game for utility-based cooperative cognitive radio networks. In Proceedings of ACM MobiHoc 2009, May 2009.

7. Han, Z., \& Poor, H. V. (2009). Coalition games with cooperative transmission: A cure for the curse of boundary nodes in selfish packet-forwarding wireless networks. IEEE Transactions on Communications, 57(1), 203-213.

8. Aram, A., Singh, C., Sarkar, S., \& Kumar, A. (2012). Cooperative profit sharing in coalition-based resource allocation in wireless networks. IEEE/ACM Transactions on Networking, 20(1), 69-83.

9. Saad, W., Han, Z., Debbah, M., Hjorungnes, A., \& Basar, T. (2009). Coalitional game theory for communication networks: A tutorial. IEEE Signal Processing Magazine, 26(5), 77-97.

10. Mathur, S., Sankaranarayanan, L., \& Mandayam, N. (2008). Coalitions in cooperative wireless networks. IEEE Journal on Selected Areas in Communications, 26(7), 1104-1115.

11. Osborne, M. \& Rubinstein, A. (1999). A course in game theory. Cambridge: The MIT Press.

12. Li, D., Xu, Y., Wang, X., \& Guizani, M. (2011). Coalitional game theoretic approach for secondary spectrum access in cooperative cognitive radio networks. IEEE Transactions on Wireless Communications, 10(3), 844-856.

13. Boyd, S. \& Vandenberghe, L. (2004). Convex optimization. Cambridge: Cambridge University Press.

14. Shapley, L. S. (1971). Cores of convex games. International Journal of Game Theory, 1(1), 11- 26.

15. Sarkar, S., Singh, C., \& Kumar, A. (2008). A coalitional game model for spectrum pooling in wireless data access networks. In Proceedings of $3 \mathrm{rd}$ information theory and applications workshop, January 2008.

16. Saad, W., Han, Z., Debbah, M., \& Hjorungnes, A. (2009). A distributed coalition formation framework for fair user cooperation in wireless networks. IEEE Transactions on Wireless Communications, 8(9), 4580-4593.

17. Fujishige, S. (1978). Polymatroidal dependence structure of a set of random variables. Information and Control, 39(1), 55-72.

18. Madiman, M. (2008). Cores of cooperative games in information theory. EURASIP Journal on Wireless Communications and Networking, 2008, 1-12

19. Cheng, H., Yang, Q., Fu, F., \& Kwak, K. S. (2011). Spectrum sharing with smooth supermodular game in cognitive radio networks. In Proceedings of 11th international symposium on communications and information technologies, October 2011.

20. Singh, C., \& Altman, E. (2011). The wireless multicast coalition game and the non-cooperative association problem. In Proceedings of IEEE INFOCOM 2011 conference, April 2011. 
21. Wu, Y., Wang, B., Liu, K. J. R., \& Clancy, T. C. (2009). Repeated open spectrum sharing game with cheat-proof strategies. IEEE Transactions on Wireless Communications, 8(4), 1922-1933.

22. Wang, B., Wu, Y., Ji, Z., Liu, K. J. R., \& Clancy, T. C. (2008). Game theoretical mechanism design methods: Suppressing cheating in cognitive radio networks. IEEE Signal Processing Magazine, 25(6), 74-84.

23. Yao, H., \& Zhong, S. (2011). Towards cheat-proof cooperative relay for cognitive radio networks. In Proceedings of ACM MobiHoc 2011, May 2011.

\section{Author Biographies}

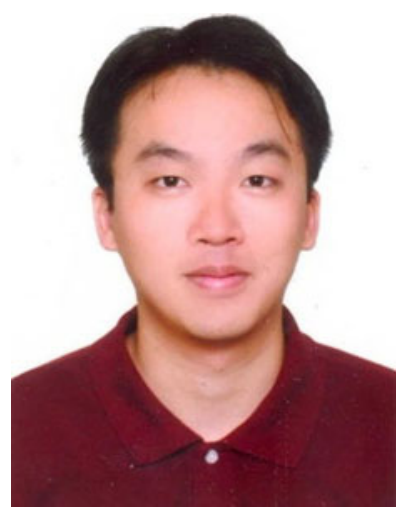

Yu-Wei Chan received the B.S. and the M.S. degree in Information Engineering from Tamkang University, Taiwan, in 1997 and 2001, respectively. He is currently pursuing the $\mathrm{Ph} . \mathrm{D}$. degree in the Department of Computer Science, National Tsing Hua University, Taiwan. His current research interests include peerto-peer computing, peer-to-peer streaming and game-theoretic resource management for distributed networks.

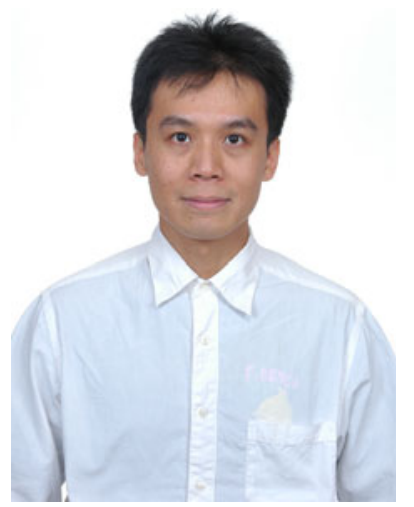

Feng-Tsun Chien received the B.S. degree from National Tsing Hua University (NTHU) in 1995, the M.S. degree from National Taiwan University (NTU) in 1997, and the Ph.D. degree from University of Southern California (USC), Los Angeles, in 2004, all in Electrical Engineering. He joined the Department of Electronics Engineering of National Chiao Tung University (NCTU), Hsinchu, in July 2005, as an Assistant Professor. His current research interests include communication systems designs, statistical signal processing, game theoretic resource allocation, and network information theory.

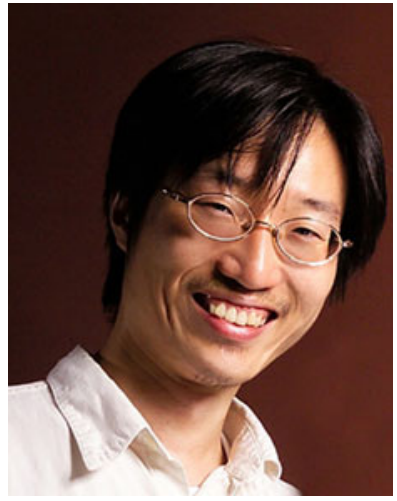

Ronald Y. Chang received the B.S. degree in electrical engineering from the National Tsing Hua University, Hsinchu, Taiwan, in 2000, the M.S. degree in electronics engineering from the National Chiao Tung University, Hsinchu, in 2002, and the $\mathrm{Ph} . \mathrm{D}$. degree in electrical engineering from the University of Southern California (USC), Los Angeles, in 2008. From 2002 to 2003, he was with the Industrial Technology Research Institute, Hsinchu. Since 2004, he has conducted research with USC as well as with the Mitsubishi Electric Research Laboratories, Cambridge, MA. Since 2010, he has been with the Research Center for Information Technology Innovation at Academia Sinica, Taipei, Taiwan, where he is now an assistant research fellow. His research interests include wireless communications and networking. He was an Exemplary Reviewer for IEEE Communications Letters in 2013, and a recipient of the Best Paper Award from IEEE Wireless Communications and Networking Conference (WCNC) 2012. He has four awarded and one pending U.S. patents.

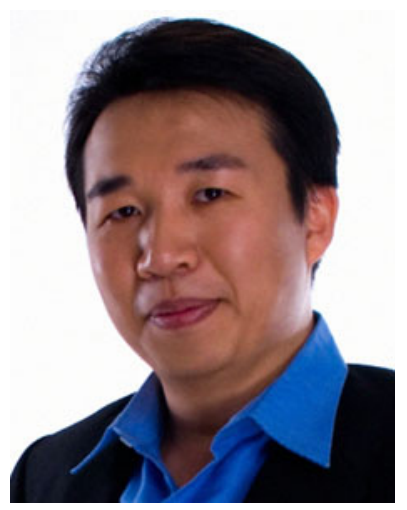

Min-Kuan Chang received B.S. degree from the National Tsing Hua University, Taiwan, in 1996, and M.S. and Ph.D. from the University of Southern California, Los Angeles, California, USA, in 1998 and 2003, respectively, all in Electrical Engineering. From 2000 to 2003, Dr. Chang was a research assistant in the Integrated Media Systems Center at the University of Southern California. In Feb. 2004, he joined the department of Electrical Engineering of National Chung Hsing University, Taichung, Taiwan. In Aug. 2012, he joined the graduate institute of Communication Engineering, where he is currently an Associate Professor. His current research interest includes power control in communication systems, wireless multimedia transmission, performance analysis and transmission design in cooperative communication systems, distributed signal processing in wireless sensor network and the game theoretical application in communication systems.

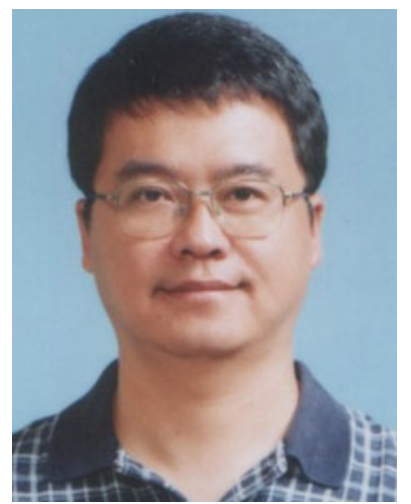

Yeh-Ching Chung received a B.S. degree in Information Engineering from Chung Yuan Christian University in 1983, and the M.S. and Ph.D. degrees in Computer and Information Science from Syracuse University in 1988 and 1992, respectively. $\mathrm{He}$ joined the Department of Information Engineering at Feng Chia University as an associate professor in 1992 and became a full professor in 1999. From 1998 to 2001, he was the chairman of the department. In 2002, he joined the Department of Computer Science at National Tsing Hua University as a full professor. His research interests include parallel and distributed processing, cluster systems, grid computing, multi-core tool chain design, and multi-core embedded systems. He is a member of the IEEE computer society and ACM. 